\title{
On Context Dependence in Modal Constructions
}

\author{
Anette Frank and Hans Kamp \\ Rank Xerox Research Centre and University of Stuttgart
}

This paper investigates a new representation format for dynamic discourse in DRT, where contextual dynamics is modeled in terms of update conditions. This new representation format is motivated by the study of context dependence in modal constructions, in particular by serious problems besetting earlier approaches to modality and modal subordination in DRT. We present an alternative DRT analysis that provides a unified analysis of relative modality and modal subordination, and which accounts for a wider range of data as regards modal subordination relative to negation and graded modal contexts.

\section{Introduction}

One of the distinguishing features of DRT is its focus on representational aspects of meaning. Another important characteristic of the theory is the insight that the meaning of sentences cannot be determined by truth conditional semantics proper. A pervasive feature of natural language semantics is that it is essentially dependent on (material introduced within) the previous discourse context; DR'T and FCS were the first semantic theories that made this specific kind of context dependence formally precise $(\operatorname{Kamp}(1981)$, Heim(1982)).

To account for anaphoric binding in donkey sentences (1a), DRT and FSC assign the conditional a 'dynamic' analysis, which is 'internal' inasmuch as it concerns the relation between the antecedent and the scope. It is 'externally static' in that the dynamically 'augmented' contexts of the antecedent and consequent are 'invisible from the outside', i.e., from the vantage point of the conditional as a whole. This accounts for the fact that the indefinite in the antecedent of the first sentence of (1a) can serve as antecedent for the pronominal in its scope, but not for the pronoun in the second sentence.

(1) a. If a farmer owns a donkey, he beats it. \# He doesn't like it.

b. If a farmer owns a donkey, he beats it. It might kick back.

Since Roberts(1989) it is well-known that there are exceptions to this rule. In Roberts' account the conditional is analyzed as externally static but internally dynamic to account for (1a), while in 'modal subordination' contexts (lb) a special accommodation device may apply, to make material that is embedded within the antecedent or scope of the conditional accessible for anaphoric binding. Geurts(1995) proposes a presuppositiorial account of modal subordination that is more restrictive than Roberts' accommodation analysis.

(C) 1997 by Anette Frank and Hans Kamp Aaron Lawson (ed), SALTVII, 151-168, Ithaca, NY: Cornell University. 
Both Roberts' and Geurts' DRT analyses are reconstructions of Kratzer's $(1978,1991)$ analysis of relative and graded modality. However, their DRS representations do not distinguish between, e.g., deontic and epistcmic modality, nor between indicative and counterfactual conditionals. Geurts' analysis does not account for modal subordination relative to a negated context, and does not appropriately render the context dependence of modal sentences that are to be interpreted as being relative to the preceding factual discourse.

We propose a new representation format for dynamic discourse in DRT, which allows us to state a unified analysis of relative modality and modal subordination that reconstructs Kratzer's notion of context dependent or relative modality at the DRS level, and thus accounts for the problems just mentioned.

\section{Context dependence in modal constructions}

There is a broad consensus nowadays that modal verbs, much like frequency adverbials, are best analyzed in terms of generalized quantification. This view not only leads to a natural analysis of graded modals (probably, unlikely), butmore importantly - it captures the inherent context dependence of modal constructions: as e.g. pointed out recently by von Fintel(1995), generalized quantifiers can be taken to involve a variable of an appropriate type, which gets interpreted in context and thus determines-in conjunction with the restrictive clause - the quantificational domain. For modal operators this view was anticipated by Kratzer(1978), who characterized modal operators as being relative to, or contextually dependent on, different kinds of intensional contexts.

\subsection{Relative and graded modality}

The impact of Kratzer's analysis of relative modality is that modal expressions, e.g. must in (2), are not semantically ambiguous between different readings traditionally classified as epistemic, deontic or circumstantial, but that there is a neutral modal operator that is contextually dependent on different kinds of intensional background contexts - epistemic (2a), deontic (2b) and circumstantial (2c) - that constitute its modal base (Kratzer(1991:639/640)).

a. (In view of the available evidence,) Jockl must be the murderer.

b. (In view of what the law provides,) Jockl must go to jail.

c. (In view of the present state of his nose etc.,) Jockl must sneeze.

Kratzer(1981) extends this analysis to the concept of graded modality, i.a. in order to account for deontic and counterfactual modality (3), where the modal operator is analyzed as doubly relative: Graded modality involves a second background context, the ordering source $o(w)$, which induces a partial order $\leq_{o(w)}$ on the set of worlds determined by the modal base $f(w)$. For the deontic 
conditional (3a), the modal operator is relative to a circumstantial modal base and a deontic ordering source, while the counterfactual ( $3 \mathrm{~b}$ ) is analyzed relative to an empty modal base and a totally realistic ordering source.

a. If Max buys a car, he must pay taxes.

b. If Max had bought a car, he would have paid taxes.

As is brought out by (4), modal subordination is to be viewed as a special instance of the more general phenomenon of relative modality: in (4a) the modal base is not given by linguistic means, and therefore must be accommodated; in (4b) the modally subordinated sentence is interpreted relative to the context set up by the scope argument of the first, modalized sentence (which in turn is to be interpreted as relative to some accommodated background context).

a. (Cleo is nominated for the first race.) Cleo might win the first race.

b. Suppose Cleo were nominated for the first race. She would certainly win (it).

\subsection{DRT accounts of modal subordination}

Kratzer's analysis of relative and graded modality is well suited to cope with anaphoric binding and presupposition projection out of modal contexts, and this is the main reason why it was 'reconstructed' in various theories of modality in the framework of DRT, as e.g. Roberts(1989) and Geurts(1995). Now, while both Roberts' and Geurts' theories are built on Kratzer's analysis of relative and graded modality, we argue that they do not treat modal subordination as a special instance of relative modality.

Roberts' accommodation account. Following Kratzer's analysis, modal operators are interpreted relative to a modal base and an ordering source, which denote sets of propositions, but are not defined in the DRS language and therefore are not represented in the DRS. The impact of Roberts' theory is to allow for accommodation of (sub)DRSs in order to account for modal subordination: in (5) the modal base of the necessity operator is further restricted by accommodation of the scope DRS of the previous modal structure into the restrictor DRS of the second, 'subordinated' modal construction.

A thief might break into the house. He would take the silver.

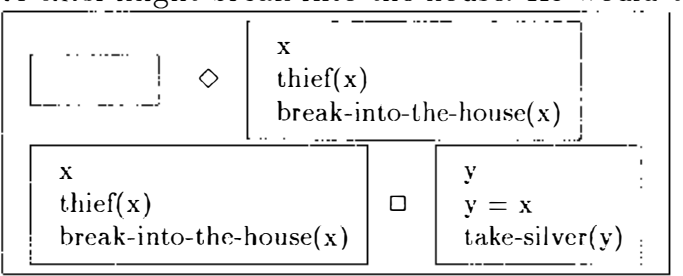


A general problem of the accommodation account is that it is too unrestricted (see Geurts(1995)). Moreover, the analysis doesn't distinguish between epistemic (5) and deontic modality (6): (6a) is assigned a DRS that is structurally identical to (5), and similarly $(3 \mathrm{a}-\mathrm{b})$ are assigned identical DRSs (modulo tense). The semantic distinction between epistemic and deontic modality is only available at the level of the verification conditions. This is unsatisfactory in particular in cases like $(6 \mathrm{~b})$, where the deontic context is introduced by linguistic means: the context dependent interpretation of the deontic sentence cannot, then, be represented in the DRS. Thus, Roberts' theory is a hybrid theory: relative modality is only captured in terms of verification conditions, while modal subordination, a special instance of relative modality, is accounted for, by the accommodation mechanism, at the level of the DRS.

(6) a. A fireman may break into the house. He must rescue the child.

b. According to German tax law, Max must pay taxes for his car.

Geurts' presuppositional/anaphoric account. Geurts' theory is also built on Kratzer's analysis, while focussing on the phenomenon of presupposition projection. It diverges from Roberts' account in that a modal is taken to presuppose its domain, thereby taming the powerful device of accommodation. The DRS language is enriched by propositional referents $p, q$, which denote sets of world-function pairs. Such referents can build terms $p+K$, "the indexed proposition denoted by $p$ incremented with the information in $K$ " (Geurts(1995:81)). Modal operators are represented as relations between propositional terms $p$ and $q$ (see (7)), and are assigned the meaning in (8), where the ordering source $o$ is a set of propositions and $\sigma, \sigma^{\prime}$ sets of world-function pairs. Modal subordination is analyzed in terms of a (variant of) the theory of presupposition as anaphora (van der Sandt(1992)): the presuppositional domain $q$ of a subordinated modal is bound to a referent $p$ established by the restrictor or scope of a preceding modal sentence. It is easily seen that a presuppositional theory of modality - in conjunction with accessibility constraints for (presuppositional) binding - is more restricted than Roberts' accommodation account.

A thief might break in. He would take the silver. Geurts(1995:86/87)

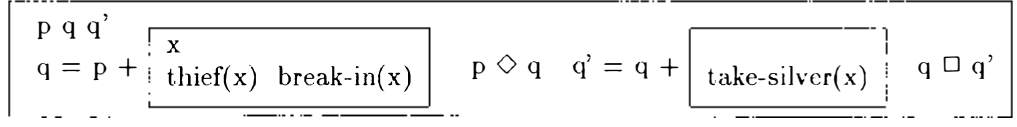

(8) Let $o$ be some given ordering source. Then:

Geurts(1995:90)

$I_{w}(\square)=\left\{\left\langle\sigma, \sigma^{\prime}\right\rangle: \forall s \in \sigma, \exists t \in \sigma, t \leq_{o(w)} s \& \forall t^{\prime} \in \sigma\right.$, if $t^{\prime} \leq_{o(w)} l$ then $\left.t^{\prime} \in \sigma^{\prime}\right\}$ $I_{w}(\diamond)=\left\{\left\langle\sigma, \sigma^{\prime}\right\rangle: \exists s \in \sigma, \forall s^{\prime} \in \sigma\right.$, if $s^{\prime} \leq_{o(u)} s$ then $\left.s^{\prime} \in \sigma^{\prime}\right\}$

But the theory suffers from two main problems. First, as in Roberts' analysis the ordering source is not represented in the DRS, but only figures in the verification condition ( 8$)$. The epistemic vs. deontic sentences $(3 a-b)$ are thus 
assigned identical DRSs. It is also not possible to represent the presuppositional/anaphorically dependent meaning of deontic sentences that are to be interpreted as being relative to a deontic antecedent context that is overtly introduced by the preceding discourse $(6 \mathrm{~b})$.

Another problem (acknowledged by Geurts) is that the analysis doesn't account for modal subordination relative to negation contexts (9). Since there is no propositional referent available that could bind the presupposed modal base of the counterfactual, the pronominal anaphor cannot be resolved. ${ }^{1}$

I don't have a microwave oven. I wouldn't know what to do with it.

This problem is of a more general nature: the analysis cannot account for relative modality wrt. the factual antecedent context, as illustrated by (10). While the presuppositional modal base is clearly dependent on the context introduced by the first sentence, there is no propositional referent that identifies this context. The modal base of the conditional can only be accommodated.

(10) There are two people in the room. If one of them leaves the room, there will still be one person in the room.

\begin{tabular}{|c|c|c|c|}
\hline $\begin{array}{l}\text { Y x p q r } \\
\text { people(Y) }|Y|=2 \quad \operatorname{room}(x) \quad \text { in }\end{array}$ & & & \\
\hline$p=? \quad q=p+\begin{array}{l}y \\
y \in Y \quad \text { leave }(y, x)\end{array}$ & $\mathrm{r}=\mathrm{q}+$ & $z \in Y \quad$ in $(z, x)$ & $\mathrm{q} \square \mathrm{r}$ \\
\hline
\end{tabular}

\section{ADRSs and the representation of contextual dynamics}

Frank(1996) pursues an analysis of modality and modal subordination that follows the spirit of Kratzer's theory of relative modality, while diverging from her analysis of deontic and counterfactual modality as involving graded modality. As in Geurts' analysis we make use of context referents $G, H$, denoting sets of world-function pairs, and which can build 'annotated' or update conditions $G:: F+K^{\prime}$. The notion of relative modality is rendered in terms of an anaphoric context referent $X^{\prime}$ that figures in the domain argument of a generalized modal quantifier. The logical form of modal operators is as in (11). Relative modality and its special instance of modal subordination are captured in terms of anaphoric binding of the modal operator's (possibly complex) modal base $X^{\prime}{ }^{2}$

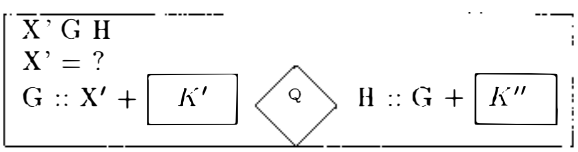

But evidently, being very similar to Geurts' approach, this anaphoric analysis doesn't solve the above mentioned problem for examples like (10) either: the DRS will not contain any context referent that represents the piece of dis- 
course introduced by the first sentence. The conditional cannot, therefore, be analyzed as anaphorically dependent on the preceding factual discourse context in terms of anaphoric binding of its modal base $X^{\prime}$.

\subsection{From relational semantics for DRSs to update conditions}

DRT's main insight is that the meaning of a sentence is essentially context dependent, that each sentence must be interpreted relative to its preceding context, or "as an addition to, or 'update' of, the context in which it is used" (van Eijck\&Kamp(1997:179)). Following this dynamic perspective, the meaning of a sentence is to be captured in terms of context change conditions rather than in terms of truth conditions proper. To make this view formally precise van Eijck\&Kamp(1997) define a relational, or dynamic semantics for DRSs, ${ }^{3}$ where the meaning of a (partial) DRS $D$ is stated as a relation between input and output assignments $s$ and $s^{\prime}$ from individuals of $U$ into $M:{ }_{s} \llbracket D \rrbracket_{s^{\prime}}^{M}$. But not only does this relational semantics account for the dynamic meaning of sentences in discourse. By extension of the DRS language with the sequencing operator ';' DRSs now explicitly represent the dynamics of discourse: ${ }_{s} \llbracket D ; D^{\prime} \rrbracket_{s^{\prime}}^{M}$ iff there is an $s^{\prime \prime}$ with ${ }_{s} \llbracket D \prod_{s^{\prime \prime}}^{M}$ and $s_{s^{\prime \prime}} \llbracket D^{\prime} \rrbracket_{s^{\prime}}^{M}$ (see van Eijck\&Kamp(1997)).

Yet, the dynamic aspect of meaning that is thus built into the semantics and representation of DRT is still of no help for the problem we encountered for (10): the states $s, s^{\prime}$ that record the assignments of referents introduced in the preceding 'input' context are not in the object language, and therefore cannot serve as representational objects to provide a context-type antecedent for the anaphoric modal base of the modal operator. But it is obvious how the dynamic view on the semantics of DRSs can be imported into the DRS syntax, so as to yield an explicit representation of this contextual dependence of sentence meaning. Modulo the intensional framework, therc is a direct correspondence between the relational semantics of DRSs and update conditions on context referents (12), where context referents denote sets of world-function pairs (see below for full definition): an update condition $G:: F+K^{\prime \prime}$ characterizes the 'update' of an 'input' context (referent) $F$ with a DRS $K^{\prime}$, to yield the 'output' context (referent) $G$, where $e(G)$ denotes the set of states $\left\langle w^{\prime}, g\right\rangle$ for which there is a state $\left\langle w^{\prime}, f\right\rangle \in e(F)$ s.th. $\left\langle w^{\prime}, f\right\rangle$ and $\left\langle w^{\prime}, g\right\rangle$ constitute correct input and output states in the relational meaning of $K^{\prime}:\left\langle w^{\prime}, f\right\rangle \backslash\left[K^{\prime} \rrbracket_{\left\langle w^{\prime}, g\right\rangle}\right.$.

$$
\langle w, e\rangle \models_{M} G:: F+K^{\prime \prime} \text { iff } e(G)=\left\{\left\langle w^{\prime}, g\right\rangle:\left(\exists\left\langle w^{\prime}, f\right\rangle \in e(F)\right)_{\left\langle u^{\prime}, f\right\rangle} \llbracket \llbracket K^{\prime} \rrbracket_{\left\langle w^{\prime}, g\right\rangle}\right\}
$$

\subsection{Representation of contextual dynamics in DRT}

Instead of using update conditions $G:: F+K^{\prime \prime}$ for 'subordinate' contcxts only, we now extend the use of these conditions to explicitly represent the dy- 
namics of a discourse within the DRS that is to represent its dynamic meaning. We will first outline the main idea by going through the critical examples, and then, in 3.4., introduce the semantic formalism in much more detail.

Let us first consider how the problematic example (10) works out within this new representation format. In (13) the first sentence, $S_{1}$, is represented as context dependent upon an antecedent context referent $F$ in terms of the update condition $G:: F+K_{1}$, where $K_{1}$ corresponds to the DRS that is to be constructed for $S_{1}$. The second sentence, $S_{2}$, is in turn characterized as context dependent on the antecedent context established by the first sentence, represented by the referent $G$, in terms of the update condition $H:: G+K_{2}$, with $K_{2}$ the representation to be constructed for $S_{2}$. Once the accessibility conditions for anaphoric binding are in place (see 3.4.), it will fall out that the context referent $G$ that represents the content conveyed by the first sentence is accessible for the anaphoric referent $X^{\prime}$, the modal base of the conditional within $K_{2}$, and that-via this anaphoric dependency - the anaphoric expressions them and the room find accessible referents, defined in the universes of $K_{\text {I }}$ and $K_{0}$. Unbounded presuppositions are accommodated into the 'highest' possible DRS, accessible from the context the presupposition projects from, here the DRS $K_{0}$ that is interpreted relative to the empty context $\Lambda$ the discourse starts out with: $e(\Lambda)=\left\{\left\langle w^{\prime}, \lambda\right\rangle: w^{\prime} \in W\right\}, \lambda$ the empty function.

(13) There are two people in the room. If one of them leaves the room, there will still be one person in the room.

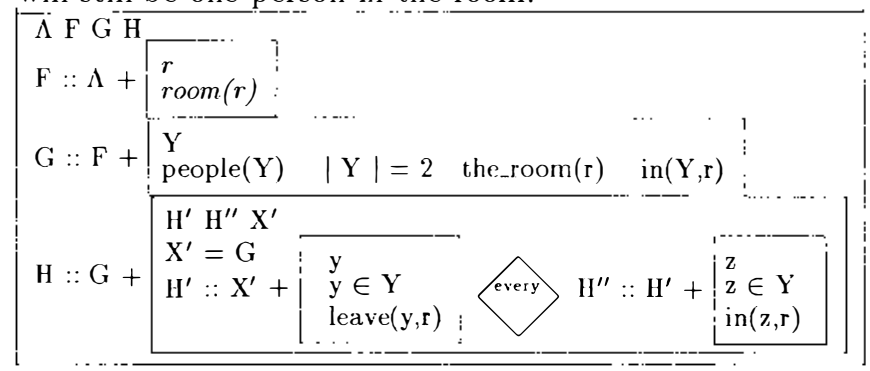

\subsection{A unified analysis of relative modality and modal subordination}

An analysis along these lines immediately accounts for modal subordination as in (14): the first conditional is represented as being relative to the (empty or accommodated) factual context $F$, enabling anaphoric or presuppositional binding into the 'main context', while the second conditional is relalive. to the modal context established by the first conditional in terms of anaphoric reference to the referent $G^{\prime \prime}$ that 'annotates $(::)$ ' its scope DRS. Accessibility must be defined so as to license binding of $X^{\prime \prime}$ to $G^{\prime \prime}$, and-via this conditionbinding of anaphoric expressions within the DRSs annotated by $H^{\prime}$ or $H^{\prime \prime}$ to material that is defined within the DRSs annotated by $G^{\prime}$ and $G^{\prime \prime}$ (see 3.4.). 
(14) If a thief breaks into the house, he will take the silver.

If in addition he finds the safe, he will try to open it.

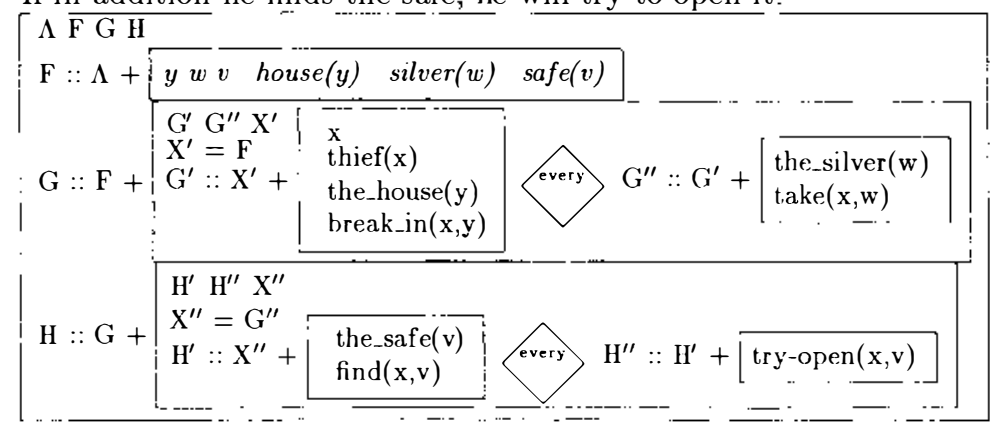

It is easy to see that this analysis caracterizes relative modality and modal subordination as a unified phenomenon: in both cases the anaphoric modal base is bound to an accessible context referent introduced by the preceding discourse. As we argued for (4), the difference between the two types of context dependent modality is captured by the fact that in the first instance (13) the antecedent referent denotes a 'factual' (nonmodal) context, whereas in the modal subordination instance of (14) the antecedent, referent denotcs a modal context, introduced by the preceding conditional.

The analysis also accounts for modal subordination relative to negated contexts (9), which was not captured by Geurts' analysis:

I didn't buy a microwave oven. I wouldn't know what to do with it.

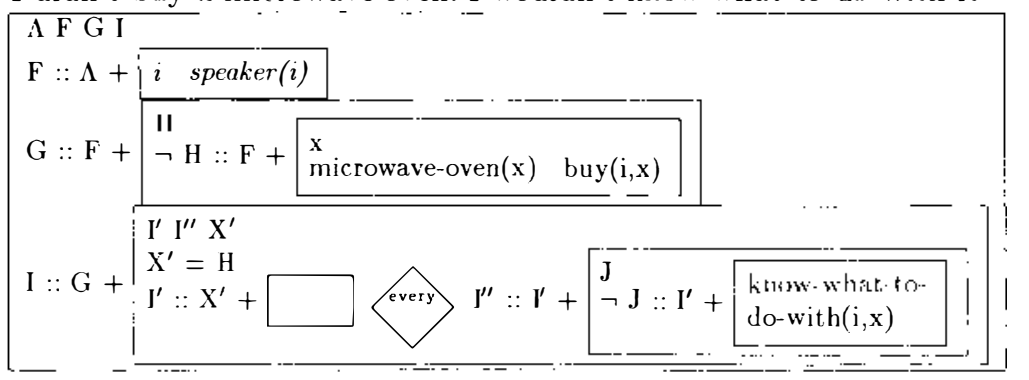

In 3.4. we define negation to take scope over an update condition. The counterfactual in (15) can then be represented as anaphoric to the referent $I I$, which denotes a counterfactual context where Max bought a microwave oven.

The analysis of deontic modals differs from Kratzer's graded modality. ${ }^{4}$ The modal base of the deontic modal is complex: it is anaphoric to a context $F+D, F$ a factual context, $D$ a context referent representing a deontic context, which is introduced by linguistic means as in (16), or else must be accommodated as obligation $(D)$. The distinction between deontic and epistemic modality is now explicitly represented, in terms of a context referent $D$ that in virtue of being an argument of a deontic predicate qualifies as deontic. 
(16) According to German tax law, Harry must pay taxes.

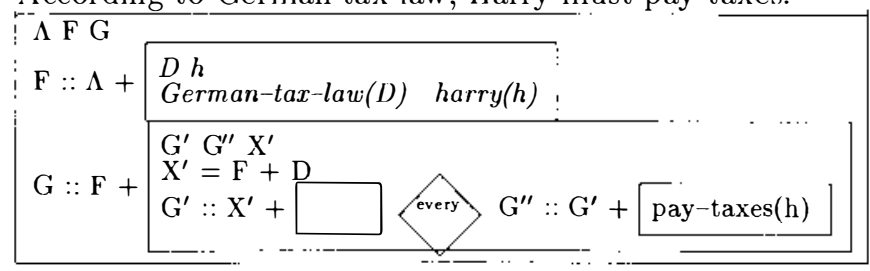

\subsection{Verification, wellfoundedness and accessibility}

We briefly present a semantics for the representations motivated above. In (21) (which is based upon a simpler language ${ }^{5}$ ) an update condition $G:: F+K^{\prime \prime}$ in (21e) not only defines a proper context change potential for $K^{\prime}$, but also constrains $K^{\prime}$ to hold true relative to the evaluation state $\langle w, e\rangle$. Two aspects require more detailed discussion: the evaluation of $K^{\prime}$ relative to $e_{\prec_{G}} \cup f$ in $(21 \mathrm{e}-\mathrm{g})$ and the normalcy restriction ${ }^{*}$ for modal quantifiers $(21 \mathrm{~g})$.

The normalcy selection function $*(w, \mathbf{G})$, to be defined in the model, denotes the set of worlds where everything holds true which is normally the case, relative to $w$, in the context $\mathrm{G}$ (cf. Morreau(1992)). In (21g) this normalcy selection function constrains the set of states in the quantificational domain, which allows us to cope with conditional variability or nonmonotonicity (sec Frank(1996)), and, moreover, to account for modal subordination relative to modal constructions hosting the quantifier no (see 4.2.).

In update conditions $G:: F+K^{\prime}(21 \mathrm{e})$ the DRS $K^{\prime}$ must be intcrpreted relative to a complex function $e_{\prec G} \cup f$ (defined by (20)). This is necessary, e.g., in order to allow for accessibility of context referents defined in $U_{K}$ from within a DRS $K^{\prime \prime}$, where $G:: F+K^{\prime \prime} \in \operatorname{Con}_{K}$. This situation is found, e.g. in (13), and is represented by the schematic DRS (17). Since $F$ is free in $K^{\prime \prime}$, the function $g$ that is to verify $K^{\prime}$ should be defined for $F$ and should assign it the value that is fixed by $e$. Since $F \in \operatorname{dom}(e)$, it seems straightforward to evaluate $K^{\prime}$ in (21e) relative to a state $e \cup f$. But this leads to the problem of non-wellfoundedness: in (17), $G \in \operatorname{dom}(e)$, in particular, $G$ denotes a set of pairs $\left\langle w^{\prime}, g\right\rangle$. If $K^{\prime}$ is evaluated relative to a state $\left\langle w^{\prime}, e \cup f\right\rangle, G$ will be in the domain of $e \cup f$, and $G$ will thus be in the domain of every $g$ in pairs $\left\langle w^{\prime}, g\right\rangle \in e(G)$ : That is, $e(G)$ would give the standard set-theoretic notion of a function belonging to its own transitive closure and thus violate the Axiom of Foundation. In other words, $\langle w, e\rangle$ does not verify $K . K$ is logically false.

$$
\begin{aligned}
& F G \\
& G:: F+X^{\prime} \\
& X^{\prime}=F
\end{aligned}
$$

To guarantee wellfoundedness we define, in (18), a relation $<$ on context referents which records the embedding structure of context referents relative to 
an embedding function $e, e=e_{1} \cup e_{2}$ where $e_{1}$ : $U_{\text {indref }-K} \rightarrow \cup_{w \in W} U_{w, M}, e_{2}$ : $U_{\text {cre } f_{-} K} \rightarrow \wp(W \times G)$ as in $(21)$. The set of context referents $\mathcal{X}$ is defined as a partially ordered system $\langle\mathcal{X},<\rangle$, which is constrained to be wellfounded (19). But note that even with (19) we still cannot assign a meaning to DRSs like (17): if $K^{\prime}$ is evaluated relative to $e \cup f, g$ will be undefined, since $G$ being in its domain immediately violates wellfoundedness as defined by (19) and (18).

$F<_{e} G$ is the smallest relation between context referents $F, G \in \mathcal{X}$ relative to an embedding function $e$ s.th.

$\forall\left\langle w^{\prime}, g\right\rangle \in e_{2}(G): F \in \operatorname{dom}\left(g_{2}\right)$ or $\forall\left\langle w^{\prime}, g\right\rangle \in e_{2}(G): \exists X \in \operatorname{dom}\left(g_{2}\right): F<_{g} X$.

(19) A function $e=e_{1} \cup e_{2}$ for ADRSs is wellfounded iff $\operatorname{dom}\left(e_{2}\right) \subseteq \mathcal{X}$ and $\langle\mathcal{X},<\rangle$ wellfounded.

In (21) wellfoundedness is therefore ensured 'on the fly', by defining $K^{\prime}$ in update conditions $G:: F+K^{\prime}$ to be evaluated relative to states $\left\langle w^{\prime}, e_{\prec G} \cup f\right\rangle$, with $e_{\prec G}$ defined in (20). Intuitively, $\operatorname{dom}\left(e_{\prec G}\right)$ is a subset of $\operatorname{dom}(e)$ which contains only those context referents $X$ that are 'smaller', in terms of the relation $<_{\circ}$ (18), than $G$, and which will thus not cause $g$ to be non-wellfounded.

(20) Let $e=e_{1} \cup e_{2}$ be a wellfounded embedding function for ADRSs, then $e_{\prec_{G}}=e_{1} \cup \epsilon_{2}^{\prime}$, where $e_{2}^{\prime}=e_{2}\left\lceil\left\{X \in \operatorname{dom}\left(e_{2}\right): X \neq G \& G \nless_{e} X\right\}\right.$.

(21) Let $K$ be a $\operatorname{DRS}\left\langle U_{K}=U_{\text {indref } K_{-}} \cup U_{\text {cref }} K_{K}, C o n_{K}\right\rangle$, with $C o n_{K}$ a set of conditions of the form referred to in $(\mathrm{a}-\mathrm{i}), \mathcal{M}$ an intensional model (see Kamp\&Reyle(1996)) and $e$ a wellfounded embedding function $e=$ $e_{1} \cup \epsilon_{2}$ where $e_{1}: U_{\text {indref }} K \rightarrow \cup_{w \in W} U_{w, M}$ and $e_{2}: U_{\text {cref- }} K \rightarrow \wp(W \times G)$ ( $G$ a set of embedding functions) and $e(\Lambda)=W \times\{\lambda\}, \lambda$ the empty function. ${ }^{*}$ is a normalcy selection function, defined in the model, which yields, for a world $w$ and a context (a set of world-function pairs) G the set of worlds $*(w, \mathbf{G})$ where everything holds true which is normally the case, relative to $w$, in the context $\mathbf{G}$ (cf. Morreau(1992)).

$\langle w, e\rangle \models_{M} K \quad$ iff $\quad \exists f: e \subseteq_{U_{K}} f \& \forall \gamma \in \operatorname{Con}_{K}:\langle w, f\rangle \models_{M} \gamma$. $\llbracket K \rrbracket_{\langle w, f\rangle}=\left\{\langle w, g\rangle: f \subseteq_{U_{K}} g \& \forall \gamma \in \operatorname{Con}_{K}:\langle w, g\rangle \models_{M} \gamma\right\}$. $\langle w, f\rangle \llbracket K \rrbracket_{\langle w, g\rangle}$ iff $f \subseteq_{U_{K}} g \& \forall \gamma \in \operatorname{Con}_{K}:\langle w, g\rangle \models_{M} \gamma$.

a. - d. atomic DRS conditions (sec Kamp\&Reyle(1993,1996))

e. $\langle w, e\rangle \models_{M} G:: F+K^{\prime} \quad$ iff $\quad e(G)=\left\{\left\langle w^{\prime}, g\right\rangle: \exists\left\langle w^{\prime}, f\right\rangle \in e(F)\right.$ s.th. $\left\langle w^{\prime}, e \prec_{G} \cup f\right\rangle \llbracket\left[K^{\prime} \rrbracket\left\langle w^{\prime}, g\right\rangle\right\} \& \exists\langle w, g\rangle \in e(G)$.

f. $\langle w, e\rangle \models_{M} \neg G:: F+K^{\prime} \quad$ iff $e(G)=\left\{\left\langle w^{\prime}, g\right\rangle: \exists\left\langle w^{\prime}, f\right\rangle \in c(F)\right.$ s.th. $\left\langle w^{\prime}, e \prec_{G} \cup \rho\right\rangle \llbracket\left[K^{\prime} \rrbracket_{\left\langle w^{\prime}, g\right\rangle}\right\} \& \neg \exists\langle w, g\rangle \in e(G)$.

g. $\langle w, e\rangle \models_{M} G:: X^{\prime}+K^{\prime}\langle\hat{\mathrm{Q}}\rangle H:: G+K^{\prime \prime} \quad$ iff $e(G)=\left\{\left\langle w^{\prime}, g\right\rangle: \exists\left\langle w^{\prime}, x^{\prime}\right\rangle \in c\left(X^{\prime}\right)\right.$ s.th. $\left.\left\langle w^{\prime}, \epsilon_{G} \cup x^{\prime}\right\rangle \llbracket K^{\prime} \rrbracket\left\langle w^{\prime}, g\right\rangle\right\} \&$ $e(H)=\left\{\left\langle w^{\prime}, h\right\rangle: \exists\left\langle w^{\prime}, g\right\rangle \in \epsilon(G)\right.$ s.th. ${ }_{\left\langle w^{\prime}, e_{\prec_{H}} \cup g\right\rangle} \llbracket\left[K^{\prime \prime} \rrbracket\left\langle w^{\prime}, h\right\rangle\right\} \&$ $\langle A, B\rangle \in \operatorname{Quant}_{M}(\mathrm{Q})$, where 


$$
\begin{aligned}
& A=\left\{\left\langle w^{\prime}, g\right\rangle:\left\langle w^{\prime}, g\right\rangle \in \dot{e}(G) \&\right. \\
& w^{\prime} \in *\left(w,\left\{\left\langle w^{\prime \prime}, g^{\prime}\right\rangle: \exists\left\langle w^{\prime \prime}, x^{\prime}\right\rangle \in e\left(X^{\prime}\right) \text { sth. }{ }_{\left\langle w^{\prime \prime}, e \alpha_{G} \cup x^{\prime}\right\rangle} \llbracket\left[K^{\prime \prime} \rrbracket_{\left\langle w^{\prime \prime}, g\right\rangle}\right\}\right)\right\} \& \\
& B=\left\{\left\langle w^{\prime}, g\right\rangle:\left\langle w^{\prime}, g\right\rangle \in e(G) \&\right. \\
& w^{\prime} \in *\left(w,\left\{\left\langle w^{\prime \prime}, g^{\prime}\right\rangle: \exists\left\langle w^{\prime \prime}, x^{\prime}\right\rangle \in e\left(X^{\prime}\right) \text { sth. }{ }_{\left\langle w^{\prime \prime}, e_{G} \cup x^{\prime}\right\rangle} \llbracket \llbracket K^{\prime} \rrbracket_{\left\langle w^{\prime \prime}, g\right\rangle}\right\}\right) \& \\
& \forall\left\langle w^{\prime}, g\right\rangle:\left\langle w^{\prime}, g\right\rangle \in e(G) \& \\
& w^{\prime} \in *\left(w,\left\{\left\langle w^{\prime \prime}, g^{\prime}\right\rangle: \exists\left\langle w^{\prime \prime}, x^{\prime}\right\rangle \in e\left(X^{\prime}\right) \text { s.th. }{ }_{\left\langle w^{\prime \prime}, e{ }_{G} \cup x^{\prime}\right\rangle} \backslash\left[K^{\prime} \rrbracket_{\left\langle w^{\prime \prime}, g\right\rangle}\right\}\right)\right. \\
& \left.\rightarrow \exists\left\langle w^{\prime}, h\right\rangle \in e(H)\right\} \text {. } \\
& \text { h. }\langle w, e\rangle \models_{M} G=F+D \quad \text { iff } \\
& e(G)=\left\{\left\langle w^{\prime}, g\right\rangle: \exists\left\langle w^{\prime}, f\right\rangle \in e(F) \exists\left\langle w^{\prime}, d\right\rangle \in e(D) \text { sth. }\left\langle w^{\prime}, g\right\rangle=\left\langle w^{\prime}, f \cup d\right\rangle\right\} \\
& \text { i. }\langle w, e\rangle \models_{M} G^{\prime} \subseteq G \text { iff } \forall\left\langle w^{\prime}, g\right\rangle \in e(G) \exists\left\langle w^{\prime}, g^{\prime}\right\rangle \in e\left(G^{\prime}\right) \text { s.th. } g^{\prime} \subseteq g{ }^{6}
\end{aligned}
$$

Kamp\&Reyle(1993) define accessibility in terms of a relation of DRS-subordination $\geq$, which largely corresponds to the hierarchical structure of DRSs. For our new DRS representation language, implicitly defined by (21), this relation is extended by the clauses in (22). (23) defines a special subordination relation $\ll$ between context referents that mirrors the semantic relation $<$ in (18). This relation further constrains the accessibility of context referents for anaphoric binding (24): a context referent $X$ may not be bound to any referent $Y$ that is 'larger' than $X$, for such bindings would be in violation of wellfoundedness.

(22) For $K_{0}^{\prime}$ a DRS, $\geq$ is the smallest relation satisfying conditions (a-d):

a. if $G:: F+K^{\prime \prime} \in \operatorname{Con}_{K_{2}} \& F:: X+K^{\prime \prime} \in \operatorname{Con}_{K_{1}}, K_{0} \geq K_{1}, K_{0}^{\prime} \geq K_{2}$ then $K^{\prime \prime} \geq K^{\prime \prime}$ and $K_{1} \geq K^{\prime \prime}$ and $K_{2} \geq K^{\prime \prime}$;

b. if $G:: F+K^{\prime \prime} \diamond H:: G+K^{\prime \prime \prime} \in \operatorname{Con}_{K_{1}}$, where $K_{0} \geq K_{1}$, then $K^{\prime \prime} \geq K^{\prime \prime \prime}, K_{1} \geq K^{\prime \prime}$ and $K_{1} \geq K^{\prime \prime \prime}$;

c. if $\neg G:: F+K^{\prime \prime} \in C o n_{K_{1}}$, where $K_{0} \geq K_{1}$, then $K_{1} \geq K^{\prime \prime}$;

d. $K^{\prime \prime} \geq K^{\prime \prime}$, and if $K^{\prime \prime} \geq K^{\prime \prime \prime}$ and $K^{\prime \prime} \geq K^{\prime \prime \prime}$ then $K^{\prime \prime} \geq K^{\prime \prime \prime}$.

(23) For $K_{0}$ a DRS, $\ll$ is the smallest relation to satisfy conditions (a-d):

a. if $G:: F+K^{\prime} \in C$ on $_{K_{1}}, K_{0} \geq K_{1}$, then $F \ll G$ and $\forall Z \in U_{K^{\prime}}: Z \ll G$;

b. if $G=F+D \in C$ on $_{K_{1}}, K_{0}^{\prime} \geq K_{1}$, then $F \ll G$ and $D \ll G$;

c. if $G^{\prime} \subseteq G \in \operatorname{Con}_{K_{1}}, K_{0} \geq K_{1}$, then $G^{\prime} \ll G$;

d. if $F \ll G$ and $G \ll H$ then $F \ll H$.

(24) A discourse referent $y \in U_{K^{\prime}}$ is accessible, within a DRS $K, K^{\prime} \geq K^{\prime \prime}$, for a discourse referent $x$ occurring in a DRS $K^{\prime \prime}$

iff $K^{\prime} \geq K^{\prime \prime}$ and if $x, y$ context referents, then $x \nless k y$.

\section{Modal subordination-what you can and cannot do}

\subsection{Negation}

Our analysis accounts for modal subordination relative to negation contexts (15), where the modal base of the subjunctive modal is anaphoric to the an- 
notating referent $H$ of the negated update condition. According to (21f) $H$ denotes a set of counterfactual states $\left\langle w^{\prime}, h\right\rangle$, where $w^{\prime} \neq w$. And from (22)(24) it follows that the referent $x$ is accessible from the scope DRS of the subordinated modal. The analysis also rules out cases like (25): the second sentence is nonmodal, and therefore cannot induce modal subordination in terms of anaphoric binding to a context referent. Since the context referent $G^{\prime}$ that is introduced by negation is not accessible as an input referent for the update condition of the second sentence, the pronoun it cannot be bound. ${ }^{7}$

Clarissa doesn't own a bike. \# She loves it.

In order to rule out subordination of indicative conditionals relative to negation contexts (26), we adopt a pragmatic constraint on sentence mood, following Stalnaker(1976): the denotation of the context referent $G^{\prime \prime}$ that annotates the restrictor of an indicative conditional must contain a state that is tied to the evaluation world. This accounts for (9/15) vs. (26): since the negated antecedent context is counterfactual (sec (21f)), indicative mood is ruled out.

(26) Fred didn't buy a microwave oven. \# He might use it.

\subsection{Graded modality}

We also account for graded modal forces (29), where for probably, unlikely, etc. we assume a probability measure $P^{8}{ }^{8}$ In $(27)$ the conditional quantifies over (sufficiently normal) worlds/states where Max goes to China, and is verified if those worlds where he buys books in China are assigned a sufficiently high relative probability. Subordination of the second sentence to the scope of the graded conditional constrains its universal quantification to range over worlds where Max buys books in China, but now with restriction to what is normally the case in such a situation. The condition is verified iff all states that pertain to such normal worlds can be extended to satisfy the scope argument.

(27) If Max goes to China, he probably buys books. Mary will admire them.

In the structurally similar (28) the modal quantifier is assigned the meaning of no, which constrains the scope argument $B$ of $Q$ to be the empty set: under normal circumstances Max will not buy books if he goes China. If the denotation of $G^{\prime}$ and $G^{\prime \prime}$ were defined in terms of the sets $A, B$ of the relational quantifier, we could not refer to $G^{\prime \prime}$ to establish a modal subordination reading: $G^{\prime \prime}$ would denote the empty set. Yet, according to (21g) $G^{\prime \prime \prime}$ denotes the full (context dependent) intension of the scope DRS, such that by anaphoric reference to $G^{\prime \prime}$ in (28) the second modal universally quantifies over those rather abnormal worlds where Max buys books in China-again with relativization to worlds where things evolve as is normal for such a (quite abnormal) situation. 
(28) If I go to China, in no case will I buy books. I wouldn't read them.

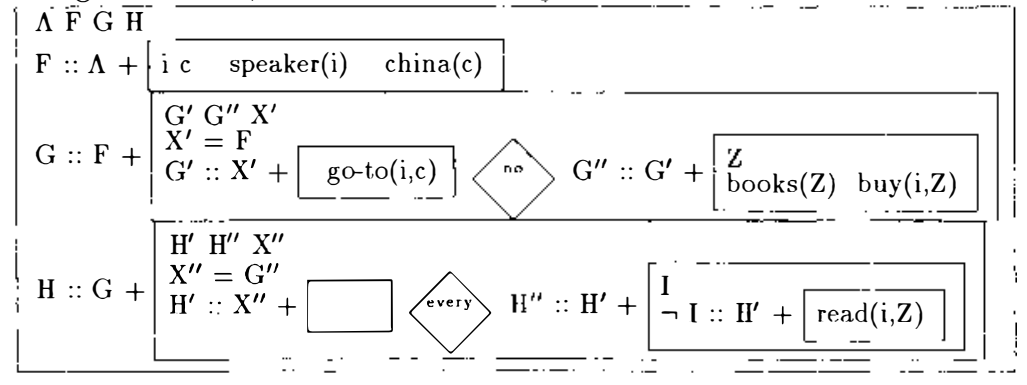

Our pragmatic constraint on sentence mood-indicative modals are relative to a context that contains a state that is tied to the evaluation world-is in accordance with $(29 \mathrm{a}-\mathrm{c}, \mathrm{f})$, but not with $(29 \mathrm{~d}-\mathrm{e}) .{ }^{9}$ While unlikely and there's a slight chance presumably both denote the quantifier unlikely, to be assigned a low relative probability, the restrictions on sentence mood differ: (29i) is a possible continuation for (29d) but not for (29e), while (29ii) is fine with (29e).

(29) a. If Max goes to China, he (necessarily) will buy a book.

b. If Max goes to China, he probably will buy a book.

c. If Max goes to China, he might buy a book.

d. If Max goes to China, there's a slight chance that he'll buy a book.

i. He will have a hard time reading it.

e. If Max goes to China, it's unlikely that he will buy a book.

f. Max goes to China, in no case will he buy a book.

i. \# He will have a hard time reading it.

ii. He would have a hard time reading it.

For indicative modals we assume the selection function ${ }^{*}$ to be centered: the evaluation world figures within the set of accessible normal worlds where Max goes to China. But with unlikely the subset of those worlds where he in addition buys books may or may not contain the evaluation world. We suggest that the contrast in (29) is to be captured in terms of a (pragmatic) restriction: for $a$ slight chance the scope $\operatorname{argument} B$ in the denotation of unlikely must, contain the evaluation world, while it may not for unlikely. These restrictions are in accordance with the monotonicity properties of these quantifiers. ${ }^{10}$ Following our pragmatic conditions on sentence mood, anaphoric reference to the scope argument $G^{\prime \prime}$ of unlikely is then only licit for (29ii), while for a slight chance modal subordination is possible with indicative mood (29i). ${ }^{11}$

\subsection{Modal subordination vs. accommodation}

We have shown that the anaphoric analysis of modal subordination copes with a wide variety of data. Yet, it is not uncontroversial that the anaphoric approach is the right way to go (see e.g. Roberts(1995)). 
Let us first mention one immediate problem of the anaphoric approach, which, however, can be resolved quite straightforwardly. (30) is semantically equivalent to (28), but structurally distinct. In (30) the context referent that represents the negated context is embedded within the conditional's scope and not accessible for the subsequent modal, to induce modal subordination.

(30) If Max goes to China, he will not buy books. He would not read them.

One could take this as a weakness of the anaphoric/presuppositional approach to modal subordination, and argue instead that the 'inherent' force of subjunctive mood is to refer to (and accommodate) the other case, computed as the complement (set) of some accessible context (see Corblin(1994)). But besides the wellformed subordination cases (30) and (9/15) this predicts (31a) to mean that Fred would have been unhappy if he hadn't got a letter! (31b) shows that (anaphoric) reference to the other case is only possible for otherwise. Instead, we propose to analyze (30) as structurally equivalent to (28), given the equivalence of $\forall \neg$ and $\neg \exists$. This can be defended in view of the syntax-semantics in terface, since the (implicit) modal quantifier and sentential negation a re both located within the functional projection of sentence structure.

$$
\text { Fred got a letter today. }
$$

a. \# He would have been unhappy.

b. Otherwise he would have been unhappy.

Roberts(1995) explicitly argues against the anaphoric approach. One of her central examples is (32), which an anaphoric analysis is unable to handle. Yet, (32) does not strike us as a particularly coherent discourse. ${ }^{12}$ It requires some additional inferencing, namely accommodation of the missing information that the leprechaun Andy could meet is one of those who have a pot of gold (or a flying carpet in (i) of fn.12). But this accommodated information doesn't correspond to the presupposed modal base of the conditional in $(32 \mathrm{c}) .{ }^{13}$

(32) a. If Andy met a leprechaun, he'd be delighted. Roberts(1995, 674)

b. Leprechauns sometimes have a pot of gold.

c. If Andy was really lucky the lepr[.] might let him have some of it.

We make a clear distinction between the anaphoric analysis of modal subordination, which may involve accommodation of the presupposed modal base if no appropriate antecedent referent is found, and accommodation of otherwise presupposed material, which may contribute to enabling anaphoric binding, but must be triggered by presuppositional elements (e.g. really lucky in (32)), or, as Roberts(1995) points out, specific contextual licensing conditions. This distinction is illustrated by (33a b) (from Corblin(1994)). Speakers uniformly affirmed that - without the bracketed material- $(33 \mathrm{~b})$ requires some further interpretation effort, as opposed to $(33 \mathrm{a})$, which comes down to 'adding', or accommodaling the information that is carried by the bracketed otherwise. 
(33) a. Mary didn't give the name of the witness.

(If she had done so/ Otherwise) They would have killed him.

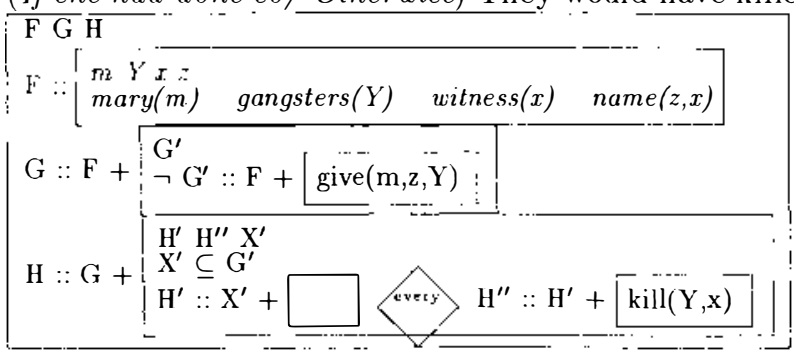

b. $\bar{M}$ ary gave the name of the witness.

(If she hadn't done so/ Otherwise) They would have killed her.

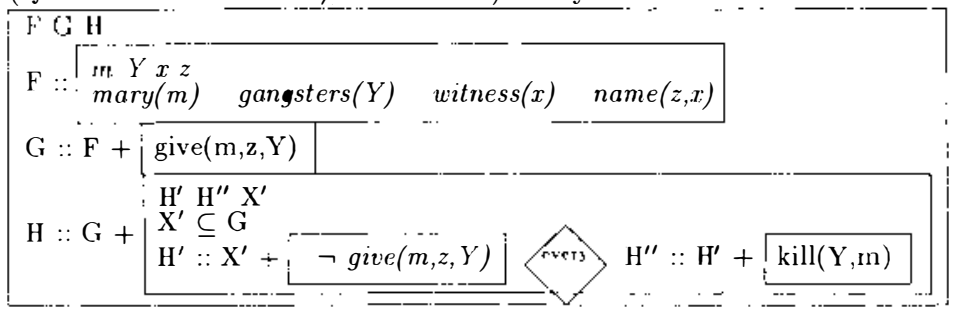

Kasper(1992) gives an analysis of simple subjunctive sentences as involving implicitly restricted counterfactual conditionals, which presuppose, in the implicit antecedent clause, "the preconditions for the possibility of the consequent to be true". In (33a), this presupposition is satisfied by modal subordination or anaphoric binding of the modal base to the context (referent) $\left({ }^{\prime}\right.$, defined by negation in the first sentence. No accommodation is in order. In (33b), by contrast - without realization of otherwise - the DRS does not contain any appropriate context referent that could instantiate the modal base of the implicitly restricted conditional, and at the same time satisfy this specific presupposition. Choosing the factual antecedent context $G$ as modal base of the counterfactual, the "preconditions for the consequent to become true" must therefore be accommodated into the restrictor DRS: the most straightforward condition that comes to mind, here, is that Mary didn't give the witness' name. It is this additional accommodation 'effort' that we believe makes (33b) slightly 'harder' to process as compared to (33a).

In sum, then, we consider examples like (31a.), (32) and (33b) to be distinct from modal subordination proper, which we conceive of as a special instance of relative modality, and which we analyzed in terms of anaphoric binding/accommodation of the modal's anaphoric modal base $X^{\prime}$. The cxamples that at first sight seem to be problematic for this 'syntactically conditioned' anaphoric approach were shown to involve accommodation, in particular, accommodation of further material, distinct from the anaphoric modal base. Since, as Roberts(1995) points out, accommodation is to be licensed pragmatically, the ease or difficulty of processing examples like (31a), (32), or (33b) will 
be heavily dependent on the particular contextual setup, or the 'willingness' of the interpreter to do accommodation more or less gratuitously. We consider it as a pro of the anaphoric approach that it captures this distinction.

\section{Conclusion}

We have given a unified analysis of relative modality and modal subordination, based on a new DRT representation format that explicitly represents the contextual dynamics of discourse. The analysis accounts for a wider range of data than previous DRT approaches: in particular, it improves over Roberts' and Geurts' reconstructions of Kratzer's theory of modality in that all relevant contextual parameters of modal constructions are represented at the level of the DRS. We have shown how to solve the problem of wellfoundedness that, was imported by use of our new representation format, and formulated syntactic constraints on anaphoric binding, which also preserve wellfoundedness.

The analysis characterizes modal subordination as an anaphoric phenomenon, a special instance of relative modality. We argue that examples that seem problematic for the anaphoric approach to modal subordination can be solved, if we clearly distinguish between modal subordination proper, which is restricted in terms of syntactic conditions on anaphoric binding, and accommodation, which is dependent on contextual and pragmatic licensing conditions.

\section{Endnotes}

* The acknowledgements of this paper address in the first place, and somewhat unusually, one of its authors, Hans Kamp, and Ede Zimmermann. The paper reports on results of a dissertation who benefitted greatly from the support and insight of these two teachers. We also would like to thank the following people for valuable comments, questions and discussion: Nicholas Asher, Miriam Butt, Tim Fernando, Josef van Genabith, Daniel Hardt, Rodger Kibble, Peter Krause, Arthur Merin, Uwe Reyle, Robert van Rooy, Daniel Rossi, Antje Roßdeutscher, Michael Schiehlen, Ben Shaer, and Carl Vogel.

${ }^{1}$ Geurts resorts to a notion of contextual suppletion in order to solve this problem. See Frank(1996) for criticism.

2 A complex modal base is used for the analysis of deontic modality (see 3.3.).

${ }^{3}$ See also Kamp\&Reyle(1996), Kamp(1996). Their definition of relational semantics follows the spirit of Heim(1982) and Groenendijk\&Stokhof(1991).

${ }^{4}$ See Frank(1996) for motivation and comprehensive analysis. The DRS given in (16) is overly simplistic: it doesn't account for a possibly conflicting factual antecedent context, and ignores problems of 'trivially' true deontic sentences. ${ }^{5}(21)$ is based on the simpler language $\mathrm{DRL}_{1}^{*}$, defined in (i) and (ii). Here and in (21) we assume Kamp\&Reyle's(1993,1996) syntax and verification conditions for atomic DRS conditions. The verification of update conditions (ii.e) 
only defines the context change potential for $K^{\prime}$ relative to $F$. Nothing follows from (ii.e) as to the truth of $K^{\prime \prime}$ relative to $\langle w, e\rangle$. Assertion of truth is defined by the truth predicate (i.f), applied to a context referent $G$. The predicate is verified by $\langle w, e\rangle$ iff there is a state $\left\langle w^{\prime}, g\right\rangle \in e(G)$ with $w^{\prime}=w$. Instead of a simple assertive DRS $K^{\prime \prime}$ we thus construct a complex structure, consisting of an update condition $G:=F+K^{\prime}$ together with the truth predicate ${ }^{\vee} G$. Yet, we refrain from using a special truth predicate in the representation language. Instead we chose the more constrained language that is implicitly defined by (21), where assertion of truth is built into the verification condition of updates.

$$
\begin{aligned}
& \text { e. } G:=F+K^{\prime \prime} \quad \text { f. } \quad{ }^{\vee} \mathrm{G} \quad \text { g. } \neg{ }^{\vee} \mathrm{G} \quad \text { h. } \quad \mathrm{G} \quad \mathrm{Q} \quad \mathrm{H} \text {. } \\
& \text { e. }\langle w, e\rangle \vDash \models_{M} G:=F^{\prime}+K^{\prime \prime} \text { iff } \\
& e(G)=\left\{\left\langle w^{\prime}, g\right\rangle: \exists\left\langle w^{\prime}, f\right\rangle \in e(F) \text { s.th. }\left\langle w^{\prime}, e_{\prec G} \cup f\right\rangle \llbracket\left[K^{\prime} \rrbracket\left\{w^{\prime}, g\right\rangle\right\} .\right. \\
& \text { f. }\langle w, e\rangle \models_{M}{ }^{\vee} G \quad \text { iff } \exists\langle w, g\rangle \in e(G) \text {. } \\
& \text { g. }\langle w, e\rangle \models_{M} \neg^{\vee} G \quad \text { iff } \neg(\exists\langle w, g\rangle \in e(G)) \text {. } \\
& \text { h. }\langle w, e\rangle \models_{M} \mathrm{G} \text { Q } \mathrm{H} \text { iff }\langle A, B\rangle \in \text { Quant }_{M}(\mathrm{Q}) \text {, where } \\
& A=\left\{\left\langle w^{\prime}, g\right\rangle:\left\langle w^{\prime}, g\right\rangle \in e(G) \& w^{\prime} \in *(w, e(G))\right\} \& \\
& B=\left\{\left\langle w^{\prime}, g\right\rangle:\left\langle w^{\prime}, g\right\rangle \in e(G) \& w^{\prime} \in *(w, e(G)) \&\right. \\
& \left.\forall\left\langle w^{\prime}, g\right\rangle:\left\langle w^{\prime}, g\right\rangle \in e(G) \& w^{\prime} \in *(w, e(G)) \rightarrow \exists\left\langle u^{\prime}, h\right\rangle \in e(H)\right\} .
\end{aligned}
$$

${ }^{6}$ Context reduction (21.i) is used for counterfactuals and deontic modality.

${ }^{7}$ Yet, we account for cases like Max doesn't own a car. So he doesn't have to park it. The input referent of the negated condition of the sccond sentence can refer to the annotating referent of the negated condition of the first sentencc. ${ }^{8}$ E.g., Quant $M($ probably $)(A, B)=\{\langle A, B\rangle: P(\operatorname{cs}(A) \cap \operatorname{cs}(B)) / P(\operatorname{cs}(A)) \geq$ $.75\}$, with probability $P$ defined for sets of worlds $c s(A), \operatorname{cs}(B)$, with $A, B$ as in $(21 \mathrm{~g})$ and $c s(\mathbf{X})=\left\{w^{\prime}:\left(\exists x^{\prime}\right)\left\langle w^{\prime}, x\right\rangle \in \mathbf{X}\right\}$.

${ }^{9}$ For indicative modals the normalcy selection function is centered. So, in all of (29a-f) the evaluation world is among the normal worlds quantified over. This predicts the indicative (29i) for (29a-c) and subjunctive (29ii) for (29f).

10 See Kibble(1996), who independently investigates modal subordination with graded modals, but doesn't take into account constraints on mood.

11 However, in (i), with modal subordination relative to the restrictor argument, indicative mood is licensed, which is predicted (see fn. 9).

(i) If I go to China, it's unlikely that I'll buy books. I'll visit monuments. ${ }^{12}$ The reason (32) might be considered as coherent could be due to the use of the kind detroting NP gold in possessive construction. Consider (i), for comparison, which is worse than (32). Speakers who accept the German equivalent of (i) suggest that it gets better if the anaphor ihn for the carpet is replaced by einen (one- anaphora), which is generally available in positions that do not allow for individual-type anaphora, and thus calls for a separate analysis.

(i) If Andy met a unicorn, he'd be delighted. Unicorns sometimes have a flying carpet. If Andy asked it kindly, the unicorn might give it to him.

13 Instead, it corresponds to the presupposition that is triggered within the conditional's antecedent, by the phrase really lucky, which is, then, responsible for the local accommodation of this 'missing' additional assumption. 


\section{References}

Corblin, F. 1994. "L'anaphore en subordination modale", in: Actes du colloque d'Anvers: Relations anaphoriques et (in)cohérence, CNRS Rennes.

van Eijck, D.J.N./ Kamp, H. 1997. "Representing Discourse in Context", in: Van Benthem, J./ Ter Meulen, A. (eds): Handbook of Logic and Linguistics, Elsevier, Amsterdam, 179-237.

von Fintel, K. 1995. "Presupposition Accommodation and Quantifier Domains", in: Prague Workshop on Context Dependence, to appear.

Frank, A. 1996. Context Dependence in Modal Constructions, Doctoral Dissertation, University of Stuttgart.

Geurts, B. 1995. Presupposing, Doctoral Dissertation, University of Stuttgart.

Groenendijk, J./ Stokhof, M. 1991. "Dynamic Predicate Logic", in: Linguistics and Philosophy, 14, 39-100.

Heim, I. 1982. The Semantics of Definite and Indefinite Noun Phrases in English, PhD Thesis, University of Massachusetts, Amherst.

Kamp, H. 1981. "A Theory of Truth and Semantic Representation", in: Groenendijk, J./ Janssen, T./ Stokhof, M. (eds): Formal Methods in the Study of Language. Amsterdam, 277-322.

Kamp, H./ Reyle, U. 1993. From Discourse to Logic, Vol.I, Dordrecht,Kluwer.

Kamp, H./ Reyle, U. 1996. From Discourse to Logic, Vol.II, ms, Stuttgart.

Kasper, W. 1992. "Presuppositions, Composition and Simple Subjunctives", in: Journal of Semantics 9, 307-331.

Kibble, R. 1996. "Modal subordination, focus and complement anaphora", in: Ginzburg,J./ Khasidashvili,Z./ Vogel,C. /Lévy,J-J./ Vallduvi,E.(eds): The Tbilisi Symposium on Language, Logic and Computation, CSII.

Kratzer, A. 1978. Semantik der Rede. Kontexttheorie - Modalwörter-Konditionalsätze. Königstein/Taunus: Scriptor.

Kratzer, A. 1981. "The Notional Category of Modality", in: Eikmeyer, J.J. \& Rieser, H. (eds): Words, Worlds, and Contexts, Berlin, 38-74.

Kratzer, A. 1991. "Modality" / "Conditionals", in: von Stechow, A./ Wunderlich, D. (eds.) Semantik. Ein internationales Handbuch der zeitgenössischen Forschung, Berlin, 639-656.

Morreau, M. 1992. Conditionals in Philosophy and Artificial Intelligence. University of Amsterdam, PhD dissertation.

Roberts, C. 1989. "Modal Subordination and Pronominal Anaphora in Discourse", in: Linguistics and Philosophy 12, 683-721.

Roberts, C. 1995. "Domain Restriction in Dynamic Semantics", in: Bach, E./ Jelinek, E./ Kratzer, A./ Partee, B.H.: Quantification in Natural Languages, Kluwer Academic Publishers, 661-700.

Stalnaker, R. 1976. "Indicative Conditionals", in: Kasher, A.: Language in Focus, Dordrecht, Reidel, 179-196.

van der Sandt, R. 1992. "Presupposition Projection as Anaphora Resolution", in Journal of Semantics 9, 333-377. 